\title{
Wireless Network Positioning Technology
}

\author{
lei Wu
}

Wuhan Institute of Shipbuilding Technology

89370742@qq.com

\begin{abstract}
Keywords: Wireless Network; Positioning Technology; Wireless Local Area Network (LAN); Fingerprint
\end{abstract}

\begin{abstract}
This paper introduces the basic concept of location information and performance analysis of location indicators, classifies the difference of all kinds of positioning methods according to the positioning principle and strategy, briefly introduces and compares the cellular networks with wireless local area network (WLAN) topology of commonly used positioning system and technology principle, makes a comparable analysis of the development status and characteristics of the WLAN based on signal strength technology, classification, characteristics and performance, and finally gives a summary and prospect of the wireless network positioning technology research and application.
\end{abstract}

\section{Introduction}

With the development of wireless communication technology and the improvement of data processing capability, location-based services become one of the most potential mobile Internet businesses. Regardless of indoor or outdoor environment, the demand for mobile terminal location information and location service is becoming increasingly urgent. Communication and positioning of two systems are mutual integrated and mutual promoted. The position of mobile terminal is determined by wireless communication and parameter measurement, and location information can be used to support position service and optimize network management, improve the service quality and network performance. Therefore, it is a hot research topic to obtain the positioning technology and positioning system of mobile position information quickly, accurately and robustly in various wireless networks.

\section{Basic Concepts of Positioning}

Mobile location relates to mobile wireless communication, mathematics, geography information and computer science, and other disciplines of knowledge, and it is easy to confuse the basic concept of some mobile positioning methods. Therefore, it is necessary to first clarify some basic concepts.

Physical and Abstract Position. The position information provided by the positioning system can be divided into two categories: the position information in the physical sense and the position information in the abstract sense. The position information in the physical sense refers to the position data in the physical or mathematical plane of the object that is positioned. For example, GPS can be measured in a building located at latitude, longitude and elevation of 50 meters. In contrast, the abstract location information can be expressed as: the building is located in the park in the woods or near the main teaching building in the campus, etc.

From the application point of view, different applications require that the location information abstraction levels are not the same, and some only need physical location information; while others require an abstract sense of location information, simple physical location information for them is transparent, or there is no significance. Of course, the physical location information can be converted and mapped to the abstract level of location information with the aid of the additional information base.

Positioning Performance Index. The relationship between the confidence level and the confidence level of the mathematical statistics is similar to the relationship between the two concepts 
and the accuracy of the position and the location accuracy. Strictly speaking, if the isolated point of a positioning system accuracy is meaningless. The typical correct description should be: A positioning system can be $95 \%$ of the probability (confidence level) issued to the positioning accuracy of $10 \mathrm{~m}$. Among them, the "95\%" is the positioning accuracy. The higher the positioning accuracy is, the lower the accuracy of the corresponding positioning is, and vice versa. The accuracy and accuracy of the positioning system can be improved by increasing the density of the positioning equipme nt or using a variety of different positioning techniques. Generally speaking, the indoor application needs to have higher accuracy than the outdoor application.

\section{Positioning technology classification}

The wireless positioning technology is measured by some parameters of the radio wave, according to the specific algorithm to determine the location of the object detected. The measurement parameters generally include the transmission time, amplitude, phase and arrival angle of the radio waves. The positioning accuracy depends on the method of measurement. From the perspective of positioning principle, the positioning technology can be roughly divided into three types: Based on the triangle relations and operations of positioning technology, based on the scene analysis of positioning technology and the relationship between neighboring positioning technologies based on.

The positioning technology according to the data obtained from the measurement, using geometric trigonometric or hyperbolic relationship of calculating the position of the object to be measured, it is the most important and most widely used as a positioning technology. The positioning technology based on triangular or hyperbolic relations can be subdivided into two kinds: the positioning technology based on distance measurement and the positioning technology based on angle measurement.

This localization technique first measures the distance between the refere nce points (A, B, C three point) of the known position (R1, R3, R2), and then the position of the object by triangulation is calculated. Specifically, there are three ways of distance measurement.

Direct Measurement.This method can measure the distance between the reference points and the object measured by physical movement and movement. For example, a robot moves its own probe, until a barrier is touched, and the distance between the probe and the probe is used as a distance parameter between itself and the obstacle.

Dissemination of Time. In the case of the transmission speed, the distance of the radio wave propagation is proportional to the time of its propagation. . The problems of this method include (a) the propagation of radio waves (in the non line of sight NLOS). The general solution is to increase the number of measurements, and to find out the measurement values of the statistical significance. (b) Clock accuracy. (c) Clock synchronization. To be involved in the same position reference point between the reference points must be guaranteed to the same time between the clocks.

\section{Cell ID Positioning Technology}

Cell ID technology is a method of localization of the simplest cellular networks, because the results of positioning terminal is terminal serving cell base station location, so that the positioning accuracy with sector size changes, and is characterized in that the fast, simple application, poor accuracy, us ually with other location using a combination, collectively referred to as based on cell ID positioning technology. This kind of localization technology is the complement and improvement of ID Cell technology. In the mobile cellular communications network, each cell is unique, and the mobile terminal is located in the corresponding ID Cell, which can be a rough determination of mobile terminal position.

Another parameter that can be used as a location in the GSM/GPRS system is the amount of time (TA), and the UMTS system is the corresponding measurement time (RTT, Round Trip Time). Both TA and RTT are time compensation (Offset Time) of base station to measure the distance between 
BTS and mobile phone, and analyze the area of mobile station. TA is a unit with the bit, and 1bit is equivalent to a distance of 550 meters; RTT in the bit as a unit, WCDMA 3.84M chip rate 1 bit is equivalent to a distance of 20 meters; TD-SCDMA $1.28 \mathrm{M}$ chip rate $1 \mathrm{bit}$ is equivalent to a distance of 60 meters. It is a simple and economical way to combine ID Cell with TA/RTT. All terminals can use this method to locate, which is one of its major advantages. However, the accuracy of this technology depends on the size of the district and the environment, and can only be used for rough positioning.

NMR (Measurement Report Network), also known as E-CGI (Cell Global Identification Enhanced), is a technology of two modes of autonomous and fingerprint positioning. This technique is enhanced by Cell ID and TA/RTT Cell ID+. NMR fingerprint off-line learning phase, terminal in determining the location of the sample points of each adjacent cell signal intensity were collecting and recording and the sample point serving cell ID, each adjacent cell signal strength and the precise position of the corresponding archive; in the on-line phase, NMR data terminal real-time measuring and collecting adjacent cells and reported to the network side database, query and the detected signal strength is most close to the sample position, as the final positioning result.

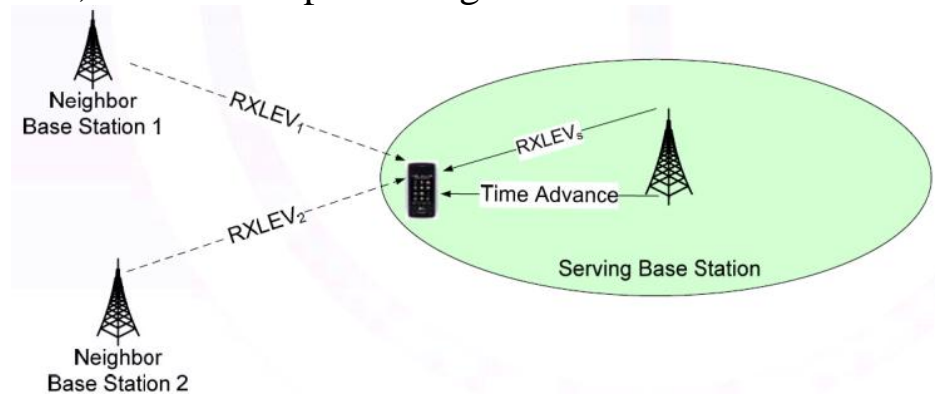

Fig. 1 NMR Neighbor Cell Measurement Positioning Principle

\section{Conclusions}

Mobile positioning technology based on CDMA network, especially based on the mobile positioning technology, is a complex technology, which involves many contents and wide range. From the perspective of mobile station positioning accuracy requirements, due to multi-path fading and multiple access interference (MAI), especially the influence of NLOS propagation, the signal characteristics measurement value always has certain deviation, so that a variety of application in characteristic measurement value error obeys zero mean Gaussian distribution of positioning algorithm, for example, the performance of minimum mean square triangle localization algorithm is unable to obtain on mobile station location of maximum likelihood estimation, and the mobile station position estimates appear larger deviations. Under the general environment of the city, with GPS as the representative of the satellite positioning technology, due to the based on high-frequency pseudo random code of precise satellite pseudo range measurements to obtain the better performance, but on the other hand, the huge downloads makes first lock time is too long. Although AGPS can greatly improve the speed of the first positioning, it still cannot completely solve the problem of poor positioning of the satellite signal condition environment. Therefore, the combination of AGPS, Cell based positioning and other technologies are more feasible and efficient choice for cellular networks.

The deployment of WLAN provides a powerful complement to the localization technology of Cell and AGPS in cellular network. Because of the same error and worse error of the feature measurement, the emphas is of the research on WLAN is based on the direction of signal intensity. Among them, the localization of fingerprint is more practical and wider than the dissemination model.

In the wireless network localization, each system and the algorithm have the ir respective characteristic and the scopes of application, which is the absolute best. Recently, the focus of the algorithm research is gradually diversified and shows a complementary trend. Each is provided with an advantage in terms of theory, strategy, network topology and field of application methods 
complementing each other, and the strength of mining is a development direction of future wireless network positioning technology.

\section{References}

[1] Rahman I. Reza, Rahman I. Reza, Co chair Dr, and Amy E. Bell. Data fusion for improved TOA/TDOA position determination in wireless systems. In Master4.pdf Song, H.L, pages 902-908, 2000.

[2] Guolin Sun and Wei Guo. A novel indoor geo-location method using MIMO array. Journal of Electronics (China), 23(6):810-813, Nov. 2006. uestcsgl@ hotmail.com.

[3]Ji Li, Ligen Wang, J. Brault, and J. Conan. Mobile location in MIMO communication systems by using learning machine. In Electrical and Computer Engineering, 2007. CCECE 2007. Canadian Conference on, pages 1066-1069, April 2007.

[4]S. J. Lee D. W. Lim. Design of an assisted gps receiver and its performance analysis. In ISCAS, 2007, pages 1742 - 1745, May 2007.

[5] SiRF. SiRFLoc Server: Wireless, A-GPS Mobile Location Software Solution.

[6] Broadcom. Budgetary pricing proposal for NMR/MRL LBS data services to CMCC corporation, 72009.

[7] IAxel Kupper. Location-based Services Fundamentals and Operations. John Wiley \& Sons, Ltd, 2005.

[8] Broadcom. Budgetary pricing proposal for NMR/MRL LBS data services to CMCC corporation, 72009. 